\title{
Treatment of Colorectal Anastomotic Leakage: Results of a Questionnaire amongst Members of the Dutch Society of Gastrointestinal Surgery
}

\author{
F. Daams ${ }^{a, b} \quad$ J.C. Slieker ${ }^{b} \quad$ A. Tedja ${ }^{a} \quad$ T.M. Karsten ${ }^{c} \quad$ J.F. Lange ${ }^{b}$

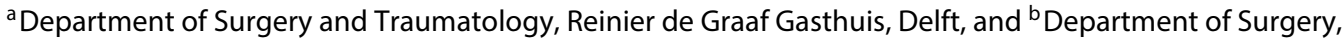 \\ Erasmus University Medical Centre, Rotterdam; ' ${ }^{\circ}$ Department of Surgery, Onze Lieve Vrouwe Gasthuis, \\ Amsterdam, The Netherlands
}

\section{Key Words}

Colorectal anastomotic leakage · Colorectal surgery .

Dutch Society of Gastrointestinal Surgery · General

peritonitis · Level of anastomosis · Local abscess · National questionnaire · Patient and surgical factors

\begin{abstract}
Anastomotic leakage after colorectal surgery is correlated with considerable morbidity and mortality. Although many studies focus on risk factors and detection, studies on the treatment strategy for colorectal anastomotic leakage are scarce. A national questionnaire amongst 350 members of the Dutch Society for Gastrointestinal Surgery was undertaken on the current treatment of colorectal anastomotic leakage. The response was $40 \%$ after two anonymous rounds. $27 \%$ of the respondents state that a leaking anastomosis above the level of the promontory should be salvaged in ASA 1-2 patients $<80$ years of age, for ASA 3 and/or $>80$ years of age this percentage is $7.3 \%$. For an anastomosis under the promontory, $50 \%$ of the respondents choose preserving the anastomosis for ASA 1-2 compared to $17 \%$ for ASA 3 and/or $>80$ years of age. In ASA 1-2 patients with a local abscess after a rectum resection without protective ileostomy, $31 \%$ of the respondents will create an protective ileostomy, $40 \%$ break down the anastomosis to create a def-
\end{abstract}

inite colostomy, in ASA 3 and/or $>80$ years of age $14 \%$ of the respondents create a protective ileostomy and $63 \%$ a definitive colostomy. In ASA 1-2 patients with peritonitis after a rectum resection with deviating ileostomy, $31 \%$ prefer a laparotomy for lavage and repair of the anastomosis, $25 \%$ for lavage without repair and $36 \%$ of the respondents prefer to break down the anastomosis. When the patient is ASA 3 and/ or $>80$ years of age, $13 \%$ prefer repair, $9 \%$ a lavage and $74 \%$ breaking down the anastomosis. This questionnaire shows that in contrast to older people, more surgeons make an effort to preserve the anastomosis in younger people.

Copyright $\odot 2013$ S. Karger AG, Basel

\section{Introduction}

Anastomotic leakage after colorectal surgery (CAL) is a major complication with a reported incidence of 2.8 $12.3 \%[1,2]$. It leads to increased morbidity (extended hospital stay, re-operation, permanent enterostomy and higher recurrence rates for carcinoma) and even up to 7\% mortality $[3,4]$. Many studies describe risk factors for CAL $[5,6]$, several studies describe prevention methods $[7,8]$, and some describe diagnostic procedures for early detection [9-12], but only a few studies have described treatment options for CAL [13-15]. In treating patients

\section{KARGER}

E-Mail karger@karger.com

www.karger.com/dsu
(C) 2013 S. Karger AG, Basel

0253-4886/12/0296-0516\$38.00/0
F. Daams

Secretariaat Chirurgie

Erasmus MC, PO Box 2040

NL-3000 CA Rotterdam (The Netherlands)

E-Mail freekdaams@gmail.com 
Table 1. Questionnaire containing multiple-choice questions on treatment strategies in CAL

1 When treating a patient with CAL would you like to be informed on the extent of leakage?

a Yes

b No

2 In your opinion would a pre-planned strategy improve outcome compared to ad hoc decision-making?

a Yes

b No

3 A deviating ileostomy is preferable above a deviating colostomy

a True

b False

4a When an intraperitoneal anastomosis is leaking in an ASA $1-2<80$ year patient this anastomosis should be preserved

a True

b False

$4 \mathrm{~b}$ When an intraperitoneal anastomosis is leaking in an ASA

$3>80$ year patient this anastomosis should be preserved

a True

b False

5a When an extraperitoneal anastomosis is leaking in an ASA

$1-2<80$ year patient this anastomosis should be preserved

a True

b False

5b When an extraperitoneal anastomosis is leaking in an ASA

$3>80$ year patient this anastomosis should be preserved

a True

b False

6a A local abscess as a presentation of a major leakage in an ASA 1-2 <80 year patient after rectal resection without deviating ostomy should be treated by

a Drainage of the abscess

b Relaparotomy, drainage of the abscess, deviating ileostomy

c Relaparotomy, drainage of the abscess, repair of the anastomosis

d Relaparotomy, drainage of the abscess, repair of the anastomosis, deviating ileostomy

e Relaparotomy, drainage of the abscess, breakdown of the anastomosis and permanent colostomy

$6 \mathrm{~b}$ A local abscess as a presentation of a major leakage in an ASA $3>80$ year patient after rectal resection without deviating ostomy should be treated by

a Drainage of the abscess

b Relaparotomy, drainage of the abscess, deviating ileostomy

c Relaparotomy, drainage of the abscess, repair of the anastomosis

d Relaparotomy, drainage of the abscess, repair of the anastomosis, deviating ileostomy

e Relaparotomy, drainage of the abscess, breakdown of the anastomosis and permanent colostomy
Table 1. (continued)

7a Faecal peritonitis from a minor leak in an extraperitoneal anastomosis with deviating ileostomy in an ASA 1-2 $<80$ year patient should be treated by

a Relaparotomy for lavage and drainage

b Relaparotomy for lavage and drainage, repair of anastomosis

c Relaparotomy for lavage, breakdown of anastomosis

$7 \mathrm{~b}$ Faecal peritonitis from a minor leak in an extraperitoneal anastomosis with deviating ileostomy in an ASA $3>80$ year patient should be treated by

a Relaparotomy for lavage and drainage

b Relaparotomy for lavage and drainage, repair of anastomosis

c Relaparotomy for lavage, breakdown of anastomosis

with anastomotic leakage, many factors should be considered before engaging a therapeutic strategy, such as patient age, co-morbidities, level of anastomosis, delay after primary operation, presence of abdominal sepsis, degree of anastomotic dehiscence. Definitions of type of leakage are not univocal [16] and, although recently the International Study Group of Rectal Cancer proposed a clinical grading, this is not widely used in the literature or in daily clinical practise [17]. Prospective randomised studies on treatment are difficult to design due to the lack of a golden standard and due to logistic problems. To overcome these problems, Phitayakorn et al. [18] used a Delphi-round to establish a treatment algorithm for CAL. In their study, the authors came to a consensus among 43 experts on colorectal surgery and radiology. The current study was undertaken simultaneously and describes the results of a questionnaire amongst all members of the Dutch Society of Gastrointestinal Surgery (NVGIC). Its goal is to reflect the surgical decision-making when facing CAL.

\section{Material and Methods}

A written questionnaire was developed by the investigators containing multiple-choice questions on treatment strategies in CAL based on clinical cases. The questionnaire can be found in table 1 . The cases were formulated to measure the effect of certain patient factors on the strategy of the surgeon treating CAL. Patient factors included anastomotic leakage above or below the level of the promontory, the percentage of anastomotic dehiscence, a primary operation with or without deviating ileostomy, ASA classification 1-2 or $>3$. A few definitions were stated in advance, such as that an anastomosis cranially to the promontory was considered to be any anastomosis after right- or left-sided colectomy and sig- 
Fig. 1. ASA $1-2$ in patients $<80$ years (left) and ASA 3 in patients $>80$ years (right).

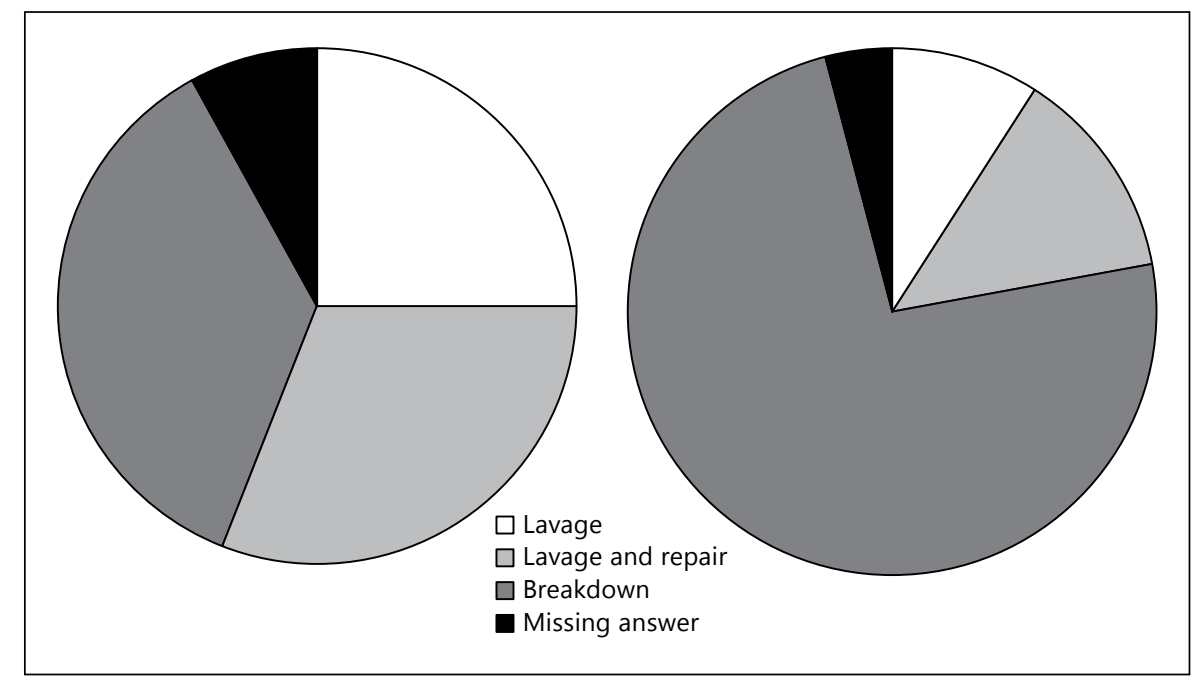

moid resection, in this article being referred to as intraperitoneal. An anastomosis caudally to the promontory was considered any anastomosis after rectum resection and is referred to as extraperitoneal. Small bowel anastomosis was not subject of this questionnaire or the ileoanal anastomosis. Furthermore, a small leak was defined as $<30 \%$ of the circumference, a large leak was defined as $>30 \%$. The questionnaire was sent to all members of the Dutch Society of Gastrointestinal Surgery by mail. At the time of sending the questionnaire, this society had 347 members, of which 53 were not practising colorectal surgery. The remaining 294 members were invited to answer the questionnaire in two anonymous rounds. Data were analysed by one investigator, who was blinded for the identity of the respondent.

\section{Results}

The response rate was $40 \%$ (137/294) over two anonymous rounds.

\section{General Considerations}

Prior to any intervention, 54\% of the responding surgeons want to be informed about the extent of anastomotic dehiscence. A majority of $72 \%$ believes that a preplanned, patient-centred strategy in treating CAL leads to a better outcome than when no strategy is followed. When a deviating enterostomy is created, $61 \%$ of the respondents create an ileostomy and 39\% a colostomy.

\section{Level of Anastomosis}

Of the respondents, $27 \%$ assume that a leaking anastomosis cranially to the level of the promontory can be preserved in ASA 1-2 patients, $<80$ years of age. For patients ASA $=/>3$ and/or $>80$ years of age this percentage is only
$7 \%$. When the anastomosis is caudally to the level of the promontory, $50 \%$ of the respondents will preserve the anastomosis in ASA $1-2$ patients and $17 \%$ for ASA $=/>3$ and/or $>80$ years of age.

\section{Local Abscess}

In ASA 1-2 patients with a local abscess with a major anastomotic leakage ( $>30 \%$ circumferential dehiscence) after rectum resection without deviating ileostomy, $60 \%$ of the respondents choose a anastomosis-sparing treatment and $40 \%$ break down the anastomosis and create a permanent colostomy. In ASA $=1>3$ and/or $>80$ years of age, these percentages are 37 and $63 \%$ respectively.

\section{General Peritonitis}

In ASA 1-2 patients with overt faecal peritonitis with a small $(<30 \%$ circumferential) dehiscence after rectum resection with a deviating ileostomy, $25 \%$ of the respondents carry out a laparotomy for peritoneal wash-out, $31 \%$ add anastomotic repair to this and $36 \%$ break down the anastomosis in addition to peritoneal wash-out. In a patient that is ASA 3 and/or $>80$ years of age, these clinical conditions render these numbers 9,13 and $74 \%$ respectively (fig. 1).

\section{Discussion}

This study shows that surgeons are to some extent influenced by patient factors and surgical factors when treating a patient with CAL. Some general considerations should be taken in mind when the results are valued. This 
questionnaire was performed amongst the members of the Dutch Society of Gastrointestinal Surgery, with a response rate of $40 \%$. Although this percentage could lead to selection bias, the response rate is similar to other nationwide questionnaires [19] and given the absolute number of 139 returned questionnaires it is unlikely that there is a strong response bias.

This study was undertaken before the International Study Group of Rectal Cancer offered a definition of CAL. Therefore, definitions used for the present study are not internationally accepted. Nevertheless, our definitions were constructed based on clinical experience and are in our opinion applicable to daily surgical practice.

This lack of standardisation might represent an explanation for the heterogeneity of the answers of the respondents. Other factors could be the different patient populations and type of hospital of the individual surgeons. Since our questionnaire was fully anonymous, no further clarification could be given on these topics. Furthermore, it can be hypothesised that some surgeons are not led by the proposed factors as age, ASA score, location of anastomosis and therefore treat all patients uniformly in case of CAL. Moreover, other factors like primary disease, immune status and timing after primary operation could be of interest in decision-making, although these factors were not the scope of this article.

\section{Patient Factors}

This study shows that the majority of the responders believe that treatment according to a personalised strategy that incorporates these patient factors leads to improved outcome. In designing a treatment strategy for CAL, patient factors are the most important input. Studies have shown that co-morbidities as diabetes, renal insufficiency, and age are on the one hand, amongst others, independent risk factors for CAL [20], but are also negative predictors for secondary peritonitis as well [21]. Contrastingly, other studies show no increased risk of complication in elderly $[22,23]$. This study shows that age and higher ASA score do influence the surgeon, leading to a tendency to preserve the anastomosis in younger patients and breakdown the anastomosis in older patients. Surgeons might have the conception that an anastomosis-sparing strategy might imply multiple re-operations, radiological interventions and delayed ICU admittance. As this leads to a serious burden for the patient's health status and requires an enormous mental effort of the patient and family, it should be conserved for younger and fitter patients. Next to this, prior to endorsing a treatment, doctors and patients should be fully committed to its accomplishment.

Current Practise in Colorectal

Anastomotic Leakage

\section{Surgical Factors}

Leakage of an intraperitoneal anastomosis is believed to be less common than after extraperitoneal anastomosis [24]. The presentation of anastomotic dehiscence varies largely from local abscesses and pelvic sepsis as a result of extraperitoneal leakage to overt abdominal sepsis as a sign of intraperitoneal leakage. Both localisation and presentation of the leak are important factors when a treatment strategy is planned. In our study, this is shown by the fact that $50 \%$ of the surgeons choose to preserve the anastomosis when it occurs as a localised extraperitoneal abscess in ASA 1-2 patients, compared to $27 \%$ of the surgeons when the anastomosis is intraperitoneal. Possibly surgeons apply other techniques for local control of extraperitoneal abscesses like radiological drainage, marsupialisation and endosponge. Although these new techniques seem promising, they also have their drawbacks $[25,26]$. These options were not offered in our questionnaire, since it is considered common surgical practice to treat a minor leak with an adjacent abscess with radiological drainage with or without diversion.

Local repair of a leaking anastomosis was condemned in the literature until some support of anastomotic preservation appeared [13,27]. Wind et al. [27] showed that of 25 patients who were re-operated for CAL, 11 were treated with preserving the anastomosis, without any sign of recurrence of leakage. In contrast to this, Rickert et al. [14] recently have shown that re-leakage occurred in 5 of 9 patients in which repair was attempted. In the same study a complete re-do of the anastomosis was successful in $84 \%$ of the patients $(2 / 12)$. In a retrospective study by Ruggiero et al. [28] for 21 out of 32 patients with CAL, a conservative treatment strategy was designed. In just 3 (14\%) of these patients, laparotomy was needed as the clinical condition worsened. The current study shows that preserving the anastomosis is considered an option by $56 \%$ of the surgeons in young patients with peritonitis. When the patient's status permits it and repair is attempted, a new anastomosis with diversion should be considered.

In a retrospective study by Paliogiannis et al. [29], early leakages (median 3.6 postoperative days) were treated more aggressively and had a greater dehiscence than leakages that became clinically apparent after a median time of 5.6 days. Furthermore, the authors found that late leakage had a milder clinical course than early leakage. When leakages appear even later, surgeons might be more reluctant to operate since dense adhesions could hamper safe dissection. Timing of diagnosis of leakage and re-opera- 
tion after the primary operation was not a factor in the questionnaire, nevertheless for the aforementioned reasons it should be taken in consideration when treating CAL.

Peritonitis due to anastomotic leakage leads to significant mortality of 6-20\% [30]. Treatment of faecal peritonitis is multidisciplinary and requires the utmost commitment of all those involved. Some studies provide guides for the surgeons when planning the surgical aspects of the treatment of abdominal sepsis. The RELAP trial has shown that an on-demand strategy for the indication of relaparotomy does not lead to increased morbidity and mortality compared to planned relaparotomy while reducing negative laparotomies and costs significantly [31]. Studies on management of anastomotic leaks show large variations in surgical procedures, with breakdown of anastomosis as the single most performed operation $[14,32]$. A recent retrospective study by Fraccalvier et al. [33] showed that anastomosis-sparing treatment by drainage and diverting loop ileostomy leads to low morbidity and mortality and high stoma reversal rates, compared to breakdown of the anastomosis. These results seem to pave the way for a randomised trial in order to rule out selection bias.
Simultaneous to this study, Phitayakorn et al. [18] performed a Delphi-round among a group of specialists, producing an algorithm for the treatment of AL. In their study, patient factors include localisation of leak, extent of anastomotic dehiscence, presence and extent of abdominal sepsis, and presence of diversion. The algorithm contains a useful combination of surgical and radiological treatment options. At several key points in the decisionmaking, such as to repair or breakdown the anastomosis at relaparotomy, the authors' algorithm leaves space for individualisation. The present study offers the current opinion amongst colorectal surgeons on those critical moments when patient characteristics are considered. It shows that patient characteristics contribute to the decision-making as young healthy patients tolerate an aggressive operative strategy in contrast to elderly patients in whom a more conservative therapy is chosen.

Concluding, this study shows that Dutch colorectal surgeons tend to preserve the anastomosis in non-septic young patients, whereas the anastomosis is broken down in older patients and/or abdominal sepsis as a rule. This study emphasizes the need for a multicentre randomised trial comparing these two strategies in colorectal anastomotic leakage.

\section{References}

$>1$ Alves AM, et al: Antibody response in mice immunized with a plasmid DNA encoding the colonization factor antigen I of enterotoxigenic Escherichia coli. FEMS Immunol Med Microbiol 1999;23:321-330.

$\checkmark 2$ Yeh CY, et al: Pelvic drainage and other risk factors for leakage after elective anterior resection in rectal cancer patients: a prospective study of 978 patients. Ann Surg 2005;241:9-13.

3 Walker KG, et al: Anastomotic leakage is predictive of diminished survival after potentially curative resection for colorectal cancer. Ann Surg 2004;240:255-259.

-4 Eriksen MT, et al: Anastomotic leakage following routine mesorectal excision for rectal cancer in a national cohort of patients. Colorectal Dis 2005;7:51-57.

$\checkmark 5$ Matthiessen P, et al: Risk factors for anastomotic leakage after anterior resection of the rectum. Colorectal Dis 2004;6:462-469.

$\checkmark 6$ Biondo S, et al: Anastomotic dehiscence after resection and primary anastomosis in leftsided colonic emergencies. Dis Colon Rectum 2005;48:2272-2280.

$>7$ Jesus EC, et al: Prophylactic anastomotic drainage for colorectal surgery. Cochrane Database Syst Rev 2004:CD002100.
-8 Tan WS, et al: Meta-analysis of defunctioning stomas in low anterior resection for rectal cancer. Br J Surg 2009;96:462-472.

$\checkmark 9$ Eckmann C, et al: Anastomotic leakage following low anterior resection: results of a standardized diagnostic and therapeutic approach. Int J Colorectal Dis 2004;19:128-133.

10 Komen N, et al: Anastomotic leakage, the search for a reliable biomarker. A review of the literature. Colorectal Dis 2008;10:109-117.

11 Den Dulk M, et al: Improved diagnosis and treatment of anastomotic leakage after colorectal surgery. Eur J Surg Oncol 2009;35: 420-426.

12 Matthiessen P, et al: Is early detection of anastomotic leakage possible by intraperitoneal microdialysis and intraperitoneal cytokines after anterior resection of the rectum for cancer? Dis Colon Rectum 2007;50:1918-1927.

13 Watson AJ, Krukowski ZH, Munro A: Salvage of large bowel anastomotic leaks. Br J Surg 1999;86:499-500.

14 Rickert A, et al: Management and outcome of anastomotic leakage after colonic surgery. Colorectal Dis 2010;12:e216-e223.

15 Verlaan T, et al: Early, minimally invasive closure of anastomotic leaks: a new concept. Colorectal Dis 2011;13(suppl 7):18-22.
16 Bruce J, et al: Systematic review of the definition and measurement of anastomotic leak after gastrointestinal surgery. Br J Surg 2001;88: 1157-1168.

17 Rahbari NN, et al: Definition and grading of anastomotic leakage following anterior resection of the rectum: a proposal by the International Study Group of Rectal Cancer. Surgery 2010;147:339-351.

18 Phitayakorn R, et al: Standardized algorithms for management of anastomotic leaks and related abdominal and pelvic abscesses after colorectal surgery. World J Surg 2008;32: 1147-1156.

19 Slieker JC, et al: Bowel preparation prior to laparoscopic colorectal resection: what is the current practice? J Laparoendosc Adv Surg Tech A 2011;21:899-903.

20 Cong ZJ, et al: Influencing factors of symptomatic anastomotic leakage after anterior resection of the rectum for cancer. World J Surg 2009;33:1292-1297.

21 McGillicuddy EA, et al: Factors predicting morbidity and mortality in emergency colorectal procedures in elderly patients. Arch Surg 2009; 144:1157-1162. 
22 Pelloni A: Colorectal surgery in patients over 80 years old. Hepatogastroenterology 2012; 59:120-123.

23 Reissman P, Agachan F, Wexner SD: Outcome of laparoscopic colorectal surgery in older patients. Am Surg 1996;62:1060-1063.

24 Platell C, et al: The incidence of anastomotic leaks in patients undergoing colorectal surgery. Colorectal Dis 2007;9:71-79.

25 Brehant O, et al: Stapled marsupialisation of chronic low rectal anastomotic sinuses. Int J Colorectal Dis 2009;24:1233-1237.

26 Arumainayagam N, Chadwick M, Roe A: The fate of anastomotic sinuses after total mesorectal excision for rectal cancer. Colorectal Dis 2009;11:288-290.
27 Wind J, et al: Laparoscopic reintervention for anastomotic leakage after primary laparoscopic colorectal surgery. Br J Surg 2007;94: 1562-1566.

28 Ruggiero R, et al: Post-operative peritonitis due to anastomotic dehiscence after colonic resection. Multicentric experience, retrospective analysis of risk factors and review of literature. Ann Ital Chir 2011;82:369-375.

29 Paliogiannis $\mathrm{P}$, et al: Conservative management of minor anastomotic leakage after open elective colorectal surgery. Ann Ital Chir 2012;83:25-28.
30 Alberts JC, Parvaiz A, Moran BJ: Predicting risk and diminishing the consequences of anastomotic dehiscence following rectal resection. Colorectal Dis 2003;5:478-482.

31 Van Ruler O, et al: Comparison of on-demand vs. planned relaparotomy strategy in patients with severe peritonitis: a randomized trial. JAMA 2007;298:865-872.

-32 Alves A, et al: Management of anastomotic leakage after nondiverted large bowel resection. J Am Coll Surg 1999;189:554-559.

33 Fraccalvier D, et al: Management of colorectal anastomotic leakage: differences between salvage and anastomotic takedown. Am J Surg 2012;204:671-676. 